\title{
FINITE INJECTIVE DIMENSION OVER RINGS WITH NOETHERIAN COHOMOLOGY
}

\author{
JEsSE BuRKe
}

\begin{abstract}
We study rings that have Noetherian cohomology over a ring of cohomology operators. Examples of such rings include commutative complete intersection rings and finite-dimensional cocommutative Hopf algebras. The main result is a criterion for a complex of modules over a ring with Noetherian cohomology to have finite injective dimension. The criterion implies in particular that for any module over such a ring, if all higher self-extensions of the module vanish, then it must have finite injective dimension. This generalizes a theorem of Avramov and Buchweitz for complete intersection rings, and a well-known theorem in the representation theory of finite groups from finitely generated to arbitrary modules.
\end{abstract}

\section{Introduction}

Let $R$ be an associative ring and $S$ a ring of cohomology operators on $R$. Thus, $S$ is a commutative graded ring and there exists a family of homogeneous maps of graded rings, indexed by complexes of $R$-modules $M$,

$$
\zeta_{M}: S \rightarrow \operatorname{Ext}_{R}^{*}(M, M)
$$

that satisfies a certain commutativity condition. See Section 3 for the full definition. We say $R$ has Noetherian cohomology over $S$ if $\operatorname{Ext}_{R}^{*}(M, M)$ is a Noetherian $S$-module via $\zeta_{M}$ for all complexes $M$, which have Noetherian cohomology over $R$.

In this paper, we prove the following:

Theorem. Let $R$ be a ring with Noetherian cohomology over a ring of cohomology operators $S$, and let $M$ be a complex of $R$-modules with $H^{n}(M)=0$ for $n \gg 0$. Let $S^{+}$be the ideal $\oplus_{i \geq 1} S^{i}$. If the $S$-module $\operatorname{Ext}_{R}^{*}(M, M)$ is $S^{+}$-torsion, then $M$ has finite injective dimension.

Recall that $\operatorname{Ext}_{R}^{*}(M, M)$ is $S^{+}$-torsion if for every $x \in \operatorname{Ext}_{R}^{*}(M, M)$ there exists an integer $n$ such that $\left(S^{+}\right)^{n} x=0$. There is, for instance, an integer $l$ depending on the degrees of the generators of $S^{+}$, such that if $\operatorname{Ext}_{R}^{n l}(M, M)=0$ for some $n \geq 1$, then $\operatorname{Ext}_{R}^{*}(M, M)$ is $S^{+}$-torsion; see 4.5. A complex has finite injective dimension if it has a bounded above semi-injective resolution; see 2.4. If the complex in question is a module, then a semi-injective resolution is an injective resolution in the classical sense. To compute $\operatorname{Ext}_{R}^{*}(M, M)$ for a complex $M$, one may use a semi-injective resolution, and so if $M$ is a module then $\operatorname{Ext}_{R}^{*}(M, M)$ agrees with the classical notion. Thus, a special case of the theorem is that if $M$ is an $R$-module with $\operatorname{Ext}_{R}^{n}(M, M)=0$ for $n \gg 0$, then $M$ has finite injective dimension.

Received by the editors October 12, 2011.

2000 Mathematics Subject Classification. xxx. 
There are many rings with Noetherian cohomology and hence to which the result above applies. First, assume that $R$ is a ring of the form $Q /\left(f_{1}, \ldots, f_{c}\right)$, where $Q$ is a commutative Noetherian regular ring of finite Krull dimension and $f_{1}, \ldots, f_{c}$ is a $Q$-regular sequence. The graded polynomial ring $S=R\left[\chi_{1}, \ldots, \chi_{c}\right]$, where the degree of each $\chi_{i}$ is 2 , is a ring of cohomology operators for $R$ and $R$ has Noetherian cohomology over $S$ by [11]. In this context, the theorem above generalizes a key instance of [2, Theorem 4.2] from finitely generated modules to a large class of complexes, including all modules:

Corollary A. Let $R=Q /\left(f_{1}, \ldots, f_{c}\right)$, where $Q$ is a commutative Noetherian regular ring of finite Krull dimension and $f_{1}, \ldots, f_{c}$ is a $Q$-regular sequence. Let $M$ be a complex of $R$-modules with $H^{n}(M)=0$ for $n \gg 0$. If $\operatorname{Ext}_{R}^{2 n}(M, M)=0$ for some $n \geq 1$, then $M$ has finite injective dimension.

Indeed, if $\operatorname{Ext}_{R}^{2 n}(M, M)=0$ for some $n$, then $\operatorname{Ext}_{R}^{*}(M, M)$ must be $S^{+}$-torsion since the degree of $\chi_{i}$ is 2 . Thus $l=2$ in the notation above; see 5.1 for further details.

Now let $R$ be a Hopf algebra over a field $k$. Any commutative subring of $\operatorname{Ext}_{R}^{*}(k, k)$ is a ring of cohomology operators on $R$; see 5.5. Let $S$ be the center of $\operatorname{Ext}_{R}^{*}(k, k)$. It follows from the main result of [10] that every finite-dimensional cocommutative Hopf algebra has Noetherian cohomology over $S$. Thus we have the following:

Corollary B. Let $R$ be a finite-dimensional cocommutative Hopf algebra over a field $k$ and let $S$ be the center of $\operatorname{Ext}_{R}^{*}(k, k)$. For an $R$-complex $M$ with $H^{n}(M)=0$ for all $n \gg 0$, if $\operatorname{Ext}_{R}^{*}(M, M)$ is $S^{+}$-torsion, then $M$ has finite injective dimension.

In particular, the result applies to the group ring of a finite group over a field where it generalizes a well-known result for finite-dimensional representations to, in particular, arbitrary representations.

For the proof of the main theorem, we work in an "infinite completion" of the bounded derived category of Noetherian $R$-modules. This allows us to avoid finiteness conditions on the complexes to which the criterion is applied. By [13], such a completion is given by the homotopy category of injective $R$-modules. We recall relevant facts about this category in Section 2. In Section 3, we give the precise definition of a ring of cohomology operators and prove a preliminary result. The proof of the main theorem occupies Section 4 and in Section 5 we apply it to the cases discussed above.

The techniques in this paper are inspired by [7]. We have minimized the use of machinery from that paper to make this one closer to being self-contained.

\section{Background}

Throughout $R$ denotes an associative ring. By " $R$-module" we mean a left-module over $R$. An $R$-complex is a complex of $R$-modules.

In this section, we briefly recall some definitions and results on triangulated categories. We then review the homological algebra of complexes that we will need.

2.1. Let $M$ be an $R$-complex. We write $\mathrm{H}^{n}(M)$ for the $n$th cohomology group of $M$ and $\mathrm{H}(M)$ for the graded $R$-module that in degree $n$ is $\mathrm{H}^{n}(M)$. We say $M$ has 
finite cohomology if $\mathrm{H}(M)$ is a Noetherian $R$-module. This implies, in particular, that $\mathrm{H}^{n}(M)=0$ for $|n| \gg 0$. The complex $M$ is acyclic if $\mathrm{H}(M)=0$.

Let $N$ be another $R$-complex. We denote the Hom-complex between $M$ and $N$ by $\operatorname{Hom}_{R}(M, N)$. This has components and differential given by

$$
\operatorname{Hom}_{R}(M, N)^{n}=\prod_{i \in \mathbb{Z}} \operatorname{Hom}_{R}\left(M^{i}, N^{i+n}\right) \quad \partial(f)=\partial^{N} \circ f-(-1)^{|f|} f \circ \partial^{M},
$$

where $|f|$ is the degree of $f$. A morphism $f: M \rightarrow N$ is a degree zero cycle of $\operatorname{Hom}_{R}(M, N)$, i.e., $|f|=0$ and $\partial(f)=0$. It is a quasi-isomorphism when $\mathrm{H}(f)$ : $\mathrm{H}(M) \rightarrow \mathrm{H}(N)$ is an isomorphism.

2.2. The homotopy category of injective $R$-modules, denoted by $\mathrm{K}(\operatorname{Inj} R)$, has as objects complexes $X$ such that $X^{i}$ is an injective $R$-module for all $i$. The morphisms between objects $X, Y$ are given by

$$
\operatorname{Hom}_{\mathrm{K}(\operatorname{Inj} \mathrm{R})}(X, Y):=\mathrm{H}^{0}\left(\operatorname{Hom}_{R}(X, Y)\right) .
$$

In other words, morphisms in $\mathrm{K}(\operatorname{Inj} \mathrm{R})$ are homotopy equivalence classes of morphisms of complexes.

The standard shift functor on $\mathrm{K}(\operatorname{Inj} \mathrm{R})$ is denoted $\Sigma$. Thus for a complex

$$
X=\cdots \rightarrow X^{n-1} \rightarrow X^{n} \rightarrow X^{n+1} \rightarrow \cdots,
$$

we have that $(\Sigma X)^{n}=X^{n+1}$ and $\partial_{\Sigma X}=-\partial_{X}$. By $\operatorname{Hom}_{\mathrm{K}}^{*}(X, Y)$ we denote the $\mathbb{Z}$-graded abelian group that in degree $n$ is $\operatorname{Hom}_{\mathrm{K}}\left(X, \Sigma^{n} Y\right)$. With multiplication given by composition $\operatorname{Hom}_{\mathrm{K}}^{*}(X, X)$ is a graded ring, whereas $\operatorname{Hom}_{\mathrm{K}}^{*}(X, Y)$ is a bimodule under the left action by $\operatorname{Hom}_{\mathrm{K}}^{*}(Y, Y)$ and the right action by $\operatorname{Hom}_{\mathrm{K}}^{*}(X, X)$.

2.3. The category $K(\operatorname{Inj} R)$ is triangulated. For a proof and reference on triangulated categories see e.g., [17]. A triangulated subcategory of $\mathrm{K}(\operatorname{Inj} \mathrm{R})$ is thick if it is closed under direct summands. It is localizing when it is closed under set-indexed direct sums. Every localizing subcategory in $\mathrm{K}(\operatorname{Inj} R)$ is automatically thick; see e.g., the proof of $[12,1.4 .8]$.

For a subclass of objects $C$ in $K(\operatorname{Inj} R)$, we denote by thick $k_{K}(C)$, respectively $\operatorname{loc}_{K}(C)$, the smallest thick, respectively localizing, subcategory containing $\mathrm{C}$. One may realize these by taking the intersection of all thick, respectively localizing, subcategories containing C.

An object $C \in \mathrm{K}(\operatorname{Inj} \mathrm{R})$ is compact if the natural map

$$
\bigoplus_{i \in I} \operatorname{Hom}_{\mathrm{K}(\operatorname{Inj} \mathrm{R})}\left(C, X_{i}\right) \rightarrow \operatorname{Hom}_{\mathrm{K}(\operatorname{Inj} \mathrm{R})}\left(C, \bigoplus_{i \in I} X_{i}\right)
$$

is an isomorphism for any set of objects $\left\{X_{i}\right\}_{i \in I}$ of $\mathrm{K}(\operatorname{Inj} \mathrm{R})$. We denote the collection of compact objects of $\mathrm{K}(\operatorname{Inj} \mathrm{R})$ by $\mathrm{K}(\operatorname{Inj} \mathrm{R})^{\mathrm{c}}$.

When $R$ is left-Noetherian, [13, 2.3.1] shows that $\mathrm{K}(\operatorname{Inj} \mathrm{R})$ is compactly generated, i.e., an object $X \in \mathrm{K}(\operatorname{Inj} \mathrm{R})$ is nonzero if and only if there exists a compact object $C \in \mathrm{K}(\operatorname{Inj} \mathrm{R})$ such that $\operatorname{Hom}_{\mathrm{K}(\operatorname{Inj} \mathrm{R})}(C, X) \neq 0$.

2.4. A complex of injective modules $I$ is semi-injective if for all acyclic complexes $A$, the complex $\operatorname{Hom}_{R}(A, I)$ is acyclic. When $I$ is semi-injective it has the following lifting property: for every morphism $\alpha: M \rightarrow I$ and every quasi-isomorphism $\beta: M \rightarrow N$ 
there exists a unique up to homotopy map $\gamma: N \rightarrow I$ making the following diagram commute:

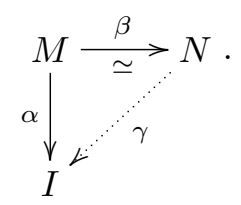

A semi-injective resolution of a complex $M$ is a quasi-isomorphism $\eta_{M}: M \rightarrow \mathrm{i} M$, where i $M$ is semi-injective. Every complex has a semi-injective resolution; this was first proven in [16]. Moreover, by the lifting property, a semi-injective resolution is unique up to isomorphism in $\mathrm{K}(\operatorname{lnj} \mathrm{R})$.

When $M$ is a module, viewed as a complex concentrated in degree 0 , a semi-injective resolution of $M$ is just an injective resolution in the usual sense.

2.5. Let $\mathrm{i} M, \mathrm{i} N$ be semi-injective resolutions of complexes $M, N$, respectively. Define the derived Hom functors as

$$
\operatorname{Ext}_{R}^{n}(M, N):=\operatorname{Hom}_{\mathrm{K}}\left(\mathrm{i} M, \Sigma^{n} \mathrm{i} N\right) \cong \mathrm{H}^{n} \operatorname{Hom}_{R}(\mathrm{i} M, \mathrm{i} N) .
$$

Set $\operatorname{Ext}_{R}^{*}(M, N)$ to be the graded $R$-module which in degree $n$ is $\operatorname{Ext}_{R}^{n}(M, N)$. The lifting property of semi-injective complexes shows that $\operatorname{Ext}_{R}^{*}(M, N)$ is independent of the choice of resolutions, up to isomorphism.

If there exists a semi-injective resolution $\eta_{M}: M \rightarrow \mathrm{i} M$ such that $(\mathrm{i} M)^{n}=0$ for all $n \gg 0$, then we say $M$ has finite injective dimension and write $\operatorname{inj} \operatorname{dim}_{R} M<\infty$.

2.6. Let $\mathrm{D}(R)$ be the unbounded derived category of $R$-modules; see e.g., [17] for the definition. We denote by $Q$ the localization functor $Q: \mathrm{K}(\operatorname{lnj} \mathrm{R}) \rightarrow \mathrm{D}(R)$ that sends a complex to its image in the derived category. When $R$ is left-Noetherian $[13,2.3 .2]$ shows that $Q$ restricts to an equivalence

$$
Q: \mathrm{K}(\operatorname{Inj} \mathrm{R})^{\mathrm{c}} \stackrel{\cong}{\longrightarrow} \mathrm{D}^{\mathrm{f}}(R)
$$

where $\mathrm{D}^{\mathrm{f}}(R)$ is the full subcategory of $\mathrm{D}(R)$ of objects with finite cohomology. By [13, $3.9]$ the functor $Q$ has a right adjoint, denoted by $Q_{\rho}$, which takes any complex to a semi-injective resolution, viewed as an object of $\mathrm{K}(\operatorname{Inj} \mathrm{R})$.

When restricted to $\mathrm{D}^{\mathrm{f}}(R), Q_{\rho}$ gives an inverse to the equivalence above. Thus the compact objects of $\mathrm{K}$ are exactly the semi-injective resolutions of objects in $\mathrm{D}^{\mathrm{f}}(R)$.

The following construction is a key part of the proof of the main theorem.

2.7. Let $\mathrm{S}=\operatorname{loc}_{\mathrm{K}}(\mathrm{C})$, for a set of compact objects $\mathrm{C}$ in $\mathrm{K}(\operatorname{Inj} \mathrm{R})$. For any object $X$ in $\mathrm{K}(\operatorname{Inj} \mathrm{R})$ there is a triangle

$$
\Gamma X \rightarrow X \rightarrow \mathrm{L} X \rightarrow
$$

such that $\Gamma X \in \mathrm{S}$ and $\mathrm{L} X \in \mathrm{S}^{\perp}$, where

$$
\mathrm{S}^{\perp}=\left\{Y \in \mathrm{K}(\operatorname{Inj} \mathrm{R}) \mid \operatorname{Hom}_{\mathrm{K}}(Z, Y)=0 \text { for all } Z \in \mathrm{S}\right\} .
$$

This is a form of Bousfield localization; see $[15,1.7]$ for a proof. 


\section{Cohomology operators}

Throughout this section $S=\oplus_{i \geq 0} S^{i}$ denotes a commutative graded ring.

3.1. We say $S$ is a ring of cohomology operators for $R$ if for every $X \in \mathrm{K}(\operatorname{Inj} \mathrm{R})$ there is a map of graded rings

$$
\zeta_{X}: S \rightarrow \operatorname{Hom}_{\mathrm{K}(\operatorname{Inj} \mathrm{R})}^{*}(X, X),
$$

such that the two $S$-module structures on $\operatorname{Hom}_{\mathrm{K}(\operatorname{Inj} \mathrm{R})}^{*}(X, Y)$ via $\zeta_{X}$ and $\zeta_{Y}$ agree. Thus, for each $\alpha \in \operatorname{Hom}_{\mathrm{K}(\operatorname{Inj} \mathrm{R})}^{*}(X, Y)$, and all homogeneous $s \in S$, we require

$$
\zeta_{Y}(s) \cdot \alpha=(-1)^{|s|} \alpha \cdot \zeta_{X}(s) .
$$

We say $R$ has Noetherian cohomology over $S$ if $S$ is a Noetherian ring of finite Krull dimension, $R$ has finite injective dimension as a left module, and $\operatorname{Hom}_{\mathrm{K}(\operatorname{Inj} \mathrm{R})}^{*}(C, C)$ is a Noetherian $S$-module for all compact objects $C$ in $\mathrm{K}(\operatorname{Inj} \mathrm{R})$.

Remark 3.2. Equivalently, $S$ is a ring of cohomology operators for $R$ if there is a ring map $S \rightarrow \mathrm{Z}(\mathrm{K}(\operatorname{Inj} \mathrm{R}))$, where $\mathrm{Z}(-)$ denotes the graded center of a triangulated category; see e.g., [7, Section 4].

A ring of cohomology operators for $R$ has been defined previously in [5] to be a ring map $S \rightarrow \mathrm{Z}(\mathrm{D}(R))$. The essentially surjective functor $\mathrm{Q}: \mathrm{K}(\operatorname{lnj} \mathrm{R}) \rightarrow \mathrm{D}(R)$ induces a ring map $\mathrm{Z}(\mathrm{K}(\operatorname{Inj} \mathrm{R})) \rightarrow \mathrm{Z}(\mathrm{D}(R))$ and thus a ring of cohomology operators in our sense gives rise to a ring of cohomology operators in the sense of [5].

In the rest of the section, we assume that $S$ is Noetherian, has finite Krull dimension, and is a ring of cohomology operators for $R$. We set $S^{+}=\oplus_{i \geq 1} S^{i}$.

We will need the following result on the structure of a ring with Noetherian cohomology.

3.3. Assume $R$ has Noetherian cohomology over $S$. Then the following hold:

(1) $R$ is left-Noetherian;

(2) inj $\operatorname{dim}_{R_{\mathfrak{p}}} R_{\mathfrak{p}}<\infty$ for all $\mathfrak{p} \in \operatorname{Spec} R^{c}$;

(3) An $R$-complex with finite cohomology $M$ has finite projective dimension if and only if $\operatorname{Ext}_{R}^{n}(M, M)=0$ for all $n \gg 0$ if and only if $M$ has finite injective dimension.

This is contained in [4], where less assumptions are placed on $S$. For the rings in Section 5 to which we apply the main theorem the properties above are well known.

The following construction was introduced in [7]:

3.4. Let $s$ be a homogeneous element of $S$ of degree $n$ and let $X$ be an object of $\mathrm{K}(\operatorname{Inj} \mathrm{R})$. The Koszul object of $s$ on $X$, denoted $X / / s$, is the mapping cone of $\zeta_{X}(s) \in$ $\operatorname{Hom}_{\mathrm{K}(\operatorname{Inj} \mathrm{R})}\left(X, \Sigma^{n} X\right)$. Thus there is an exact triangle

$$
X \stackrel{\zeta_{X}(s)}{\longrightarrow} \Sigma^{n} X \rightarrow X / / s \rightarrow
$$

and $X / / s$ is unique up to isomorphism. For $\mathrm{s}=s_{1}, \ldots, s_{r}$ a sequence of homogeneous elements of $S$, the Koszul object of s on $X$, denoted $X / / \mathrm{s}$, is defined inductively as the Koszul object of $s_{r}$ on $X / /\left(s_{1}, \ldots, s_{r-1}\right)$. 
Let $Y$ be another object of $\mathrm{K}(\operatorname{Inj} \mathrm{R})$. We need the following properties of Koszul objects:

(1) If $X$ is compact, then so is $X / / \mathrm{s}$; this follows by induction and the triangle (3.2) above.

(2) There exists an integer $n \geq 0$, independent of $X$ and $Y$, such that

$$
(\mathrm{s})^{n} \operatorname{Hom}_{\mathrm{K}(\operatorname{Inj} \mathrm{R})}^{*}(Y, X / / \mathrm{s})=0=(\mathrm{s})^{n} \operatorname{Hom}_{\mathrm{K}(\operatorname{Inj} \mathrm{R})}^{*}(X / / \mathrm{s}, Y),
$$

where $(\mathrm{s})=\left(s_{1}, \ldots, s_{n}\right)$ is the ideal in $S$ generated by $s_{1}, \ldots, s_{n}$.

(3) If $\operatorname{Hom}_{\mathrm{K}(\operatorname{Inj} \mathrm{R})}^{*}(X / / \mathrm{s}, Y)=0$ and the $S$-module $\operatorname{Hom}_{\mathrm{K}(\operatorname{Inj} \mathrm{R})}^{*}(X, Y)$ is s-torsion then

$$
\operatorname{Hom}_{\mathrm{K}(\operatorname{Inj} \mathrm{R})}^{*}(X, Y)=0 .
$$

The last two results are contained in $[7,5.11]$.

The next result shows that every compact object of $K(\operatorname{Inj} R)$ can be cut down to an object with finite projective dimension using the above construction.

Proposition 3.5. Assume $R$ has Noetherian cohomology over $S$. Let $\mathrm{s}=s_{1}, \ldots, s_{r}$ be a set of generators of the ideal $S^{+}=\oplus_{i>0} S^{i}$ and let $\mathrm{i} R \in \mathrm{K}(\operatorname{Inj} \mathrm{R})$ be an injective resolution of $R$. For every compact object $C$ of $\mathrm{K}(\operatorname{Inj} \mathrm{R})$ the object $C / / \mathrm{s}$ is in $\operatorname{thick}_{\mathrm{K}}(\mathrm{i} R)$. In particular there is an inclusion of subcategories:

$$
\operatorname{thick}_{\mathrm{K}}\left(C / / \mathrm{s} \mid C \in \mathrm{K}(\operatorname{Inj} \mathrm{R})^{\mathrm{c}}\right) \subseteq \operatorname{thick}_{\mathrm{K}(\operatorname{Inj} \mathrm{R})}(\mathrm{i} R) .
$$

Proof. By 3.4(2) there exists $n \geq 1$ such that $(\mathrm{s})^{n} \operatorname{Hom}_{\mathrm{K}(\operatorname{Inj} \mathrm{R})}^{*}(C / / \mathrm{s}, C / / \mathrm{s})=0$. Since $C / / \mathrm{s}$ is compact, the $S$-module $\operatorname{Hom}_{\mathrm{K}(\operatorname{Inj} \mathrm{R})}^{*}(C / / \mathrm{s}, C / / \mathrm{s})$ is finitely generated by the definition of Noetherian cohomology. A standard argument now shows that

$$
\operatorname{Hom}_{\mathrm{K}(\operatorname{Inj} \mathrm{R})}^{m}(C / / \mathrm{s}, C / / \mathrm{s})=0 \text { for } m \gg 0 .
$$

Since $C / / \mathrm{s}$ is compact, by 2.6 , the complex $C / / \mathrm{s}$ is semi-injective. Thus,

$$
\operatorname{Hom}_{\mathrm{K}(\operatorname{Inj\mathrm {R}})}^{*}(C / / \mathrm{s}, C / / \mathrm{s}) \cong \operatorname{Ext}_{R}^{*}(C / / \mathrm{s}, C / / \mathrm{s}) \text {. }
$$

Now 3.3 and $3.3(3)$ show that $C / / \mathrm{s}$ has finite projective dimension. One checks, by induction on projective dimension for instance, that this implies that $C / / \mathrm{s} \in$ thick $_{\mathrm{D}(R)}(R)$. Since triangulated functors preserve thick subcategories we have that

$$
Q_{\rho}(C / / \mathrm{s}) \in \operatorname{thick}_{\mathrm{K}(\operatorname{Inj} \mathrm{R})}\left(Q_{\rho} R\right) .
$$

As semi-injective resolutions are unique in $\mathrm{K}(\operatorname{Inj} \mathrm{R})$ and $C / / \mathrm{s}$ and $Q_{\rho}(C / / \mathrm{s})$ are semiinjective, we have that $Q_{\rho}(C / / \mathrm{s}) \cong C / / \mathrm{s}$ and $Q_{\rho} R \cong \mathrm{i} R$. Stringing together the above shows that $C / / \mathrm{s}$ is in $\operatorname{thick}_{\mathrm{K}}(\mathrm{i} R)$.

\section{Finite injective dimension}

In this section, we prove the theorem in the introduction. To do this we need the following:

Proposition 4.1. Let $R$ be a left-Noetherian ring that has finite injective dimension as a left $R$-module and let $M$ be an $R$-complex with $\mathrm{H}^{n}(M)=0$ for $n \gg 0$. Let $\mathrm{i} R$ 
and $\mathrm{i} M$ be semi-injective resolutions of $R$ and $M$, respectively. If $\mathrm{i} M$ is in $\operatorname{loc}_{\mathrm{K}}(\mathrm{i} R)$, then $M$ has finite injective dimension.

Proof. Since $M$ has right-bounded cohomology, we may pick a projective resolution $P \stackrel{\simeq}{\longrightarrow} M$, i.e., a quasi-isomorphism such that $P^{j}$ is projective and $P^{j}=0$ for $j \gg 0$. Each $P^{j}$ has finite injective dimension bounded by the injective dimension of the ring, which we denote by $d$.

Fix an injective resolution of each $P^{j}$ of length at most $d$. By the comparison theorem there are maps between the resolutions which form a bicomplex. Taking the total sum complex of this bicomplex gives a complex $L$ and a quasi-isomorphism $P \stackrel{\simeq}{\longrightarrow} L$, such that each $L^{j}$ is injective and $L^{j}=0$ for $j \gg 0$. Now let $L \rightarrow \mathrm{i} L$ be a semi-injective resolution. We have a diagram

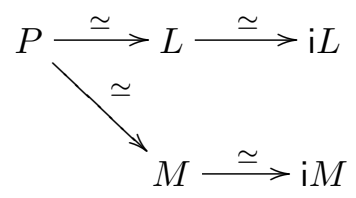

By the lifting property of semi-injective resolutions, described in 2.4 , we see that $\mathrm{i} M \cong$ $\mathrm{i} L$ in $\mathrm{K}(\operatorname{Inj} \mathrm{R})$. In particular $\mathrm{i} L$ is a semi-injective resolution of $M$ and $\mathrm{i} L \in \operatorname{loc}_{\mathrm{K}}(\mathrm{i} R)$.

Let $T$ be the mapping cone of $L \rightarrow \mathrm{i} L$. We have a triangle

$$
L \rightarrow \mathrm{i} L \stackrel{v}{\rightarrow} T \rightarrow
$$

in $\mathrm{K}(\operatorname{Inj} \mathrm{R})$. Note that $T$ is acyclic since $L \rightarrow \mathrm{i} L$ is a quasi-isomorphism. Thus, we have isomorphisms

$$
\operatorname{Hom}_{\mathrm{K}}^{*}(\mathrm{i} R, T) \cong \operatorname{Hom}_{\mathrm{K}(R)}^{*}(R, T) \cong \mathrm{H}^{*}(T)=0 .
$$

The first is $[13,2.1]$, the second is clear, and the third is the fact that $T$ is acyclic.

The full subcategory whose objects are

$$
\left\{X \mid \operatorname{Hom}_{\mathrm{K}}^{*}(X, T)=0\right\}
$$

is a localizing subcategory of $\mathrm{K}(\operatorname{Inj} \mathrm{R})$. Thus, since $\mathrm{i} R$ is in this subcategory, so is $\operatorname{loc}_{\mathrm{K}}(\mathrm{i} R)$. In particular $\mathrm{i} L \in \operatorname{loc}_{\mathrm{K}}(\mathrm{i} R)$, and thus $\operatorname{Hom}_{\mathrm{K}}^{*}(\mathrm{i} L, T)=0$. This shows that the map $v$ above is nullhomotopic. We will show that this forces $\mathrm{i} L$ to have an injective cokernel in a high degree.

Since $v$ is nullhomotopic there exists a map $s: \mathrm{i} L \rightarrow T$ such that $\partial s+s \partial=v$. Let $k$ be an integer such that $L^{n}=0$ for all $n \geq k$, which exists by assumption. Thus $v^{n}$ is bijective for all $n \geq k$ and we have that $\left(v^{n}\right)^{-1} \partial s+\left(v^{n}\right)^{-1} s \partial=1_{\mathrm{i} L^{n}}$. One checks that $v^{-1}$ commutes with the differentials in the degrees for which it is defined; this gives

$$
\partial\left(v^{n-1}\right)^{-1} s+\left(v^{n}\right)^{-1} s \partial=1_{i L^{n}} .
$$

Thus $v^{-1} s$ is a contracting homotopy of $1_{\mathrm{i} M}$ in high degrees. A simple diagram chase now shows that $\operatorname{Im}\left(\partial^{k}\right)$ splits as a submodule of $(\mathrm{i} L)^{k+1}$ and hence is injective.

Since $v$ is a bijection in degrees $n \geq k$ and $T$ is acyclic, this implies that $\mathrm{H}^{n}(\mathrm{i} L)=0$ for $n \geq k$. Thus i $L$ has an injective cokernel in a degree higher than its last nonzero cohomology; by [3, 2.4.I] this implies that $M$ has finite injective dimension. One may also verify this directly by noting that we have shown that $\mathrm{i} L \cong X \oplus Y$ with $X^{i}=0$ for $i \gg 0$ and $Y$ nullhomotopic. 
Theorem 4.2. Let $R$ be an associative ring and $S$ a Noetherian graded ring of finite Krull dimension. Assume that $S$ is a ring of cohomology operators on $R$ and that $R$ has Noetherian cohomology over $S$. For an $R$-complex $M$ with $\mathrm{H}^{n}(M)=0$ for $n \gg 0$, if the $S$-module $\operatorname{Ext}_{R}^{*}(M, M)$ is $S^{+}=\oplus_{i \geq 1} S^{i}$-torsion, then $M$ has finite injective dimension.

Proof. Let $X=\mathrm{i} M$ be a semi-injective resolution of $M$. Then, by 2.5 ,

$$
\operatorname{Ext}_{R}^{*}(M, M) \cong \operatorname{Hom}_{\mathrm{K}(\operatorname{Inj} \mathrm{R})}^{*}(X, X) .
$$

Let $\mathrm{s}$ be a finite set of generators of the ideal $S^{+}$. By 3.3, $R$ is left-Noetherian and it has finite injective dimension by the assumption of Noetherian cohomology. Thus by 4.1 it is enough to show that $\mathrm{i} M \in \operatorname{loc}_{\mathrm{K}}(\mathrm{i} R)$. Since every localizing subcategory in $\mathrm{K}(\operatorname{Inj} \mathrm{R})$ is thick (see 2.3), Proposition 3.5 shows that

$$
\operatorname{loc}_{\mathrm{K}}\left(C / / \mathrm{s} \mid C \in \mathrm{K}^{\mathrm{c}}\right) \subseteq \operatorname{loc}_{\mathrm{K}}(\mathrm{i} R) .
$$

Thus to prove the theorem it is enough to show that $X \in \operatorname{loc}_{\mathrm{K}}\left(C / / \mathrm{s} \mid C \in \mathrm{K}(\operatorname{Inj} \mathrm{R})^{\mathrm{c}}\right)$. Let us set $\mathrm{C}:=\operatorname{loc}_{\mathrm{K}}\left(C / / \mathrm{s} \mid C \in \mathrm{K}(\operatorname{Inj} \mathrm{R})^{\mathrm{c}}\right)$.

Fix a compact object $D$. By hypothesis $\operatorname{Hom}_{\mathrm{K}(\operatorname{Inj} \mathrm{R})}^{*}(X, X)$ is $S^{+}$-torsion. By the definition of cohomology operators, the action of $S$ on $\operatorname{Hom}_{\mathrm{K}(\operatorname{Inj} \mathrm{R})}^{*}(D, X)$ factors through $\operatorname{Hom}_{\mathrm{K}(\operatorname{Inj} \mathrm{R})}^{*}(X, X)$ and hence $\operatorname{Hom}_{\mathrm{K}(\operatorname{Inj} \mathrm{R})}^{*}(D, X)$ is also $S^{+}$-torsion.

Now consider the full subcategory $\mathrm{T}$ of $\mathrm{K}(\operatorname{Inj} \mathrm{R})$ with objects those $Z \in \mathrm{K}(\operatorname{Inj} \mathrm{R})$ such that $\operatorname{Hom}_{\mathrm{K}(\operatorname{Inj} \mathrm{R})}^{*}(D, Z)$ is $S^{+}$-torsion. It is clearly closed under suspension. Given a triangle $Y \rightarrow Z \rightarrow W \rightarrow \Sigma Y$ in $\mathrm{K}(\operatorname{Inj} \mathrm{R})$, there is an exact sequence of $S$-modules:

$$
\operatorname{Hom}_{\mathrm{K}(\operatorname{Inj} \mathrm{R})}^{*}(D, Y) \rightarrow \operatorname{Hom}_{\mathrm{K}(\operatorname{Inj} \mathrm{R})}^{*}(D, Z) \rightarrow \operatorname{Hom}_{\mathrm{K}(\operatorname{Inj} \mathrm{R})}^{*}(D, W) .
$$

From this we see that if $\operatorname{Hom}_{\mathrm{K}(\operatorname{Inj} \mathrm{R})}^{*}(D, Y)$ and $\operatorname{Hom}_{\mathrm{K}(\operatorname{Inj} \mathrm{R})}^{*}(D, W)$ are $S^{+}$-torsion then $\operatorname{Hom}_{\mathrm{K}(\operatorname{Inj} \mathrm{R})}^{*}(D, Z)$ is as well. This shows that $\mathrm{T}$ is triangulated. For a family of objects $\left\{Z_{i}\right\}$ in $\mathrm{T}$, we have that

$$
\operatorname{Hom}_{\mathrm{K}(\operatorname{Inj} \mathrm{R})}^{*}\left(D, \bigoplus_{i} Z_{i}\right) \cong \bigoplus_{i} \operatorname{Hom}_{\mathrm{K}(\operatorname{Inj} \mathrm{R})}^{*}\left(D, Z_{i}\right)
$$

since $D$ is compact. Thus $\mathrm{T}$ is closed under direct sums and hence is localizing. By $3.4(2)$, for every object $C$ the module $\operatorname{Hom}_{\mathrm{K}(\operatorname{Inj} \mathrm{R})}^{*}(D, C / / \mathrm{s})$ is $S^{+}$-torsion. Thus

$$
\mathrm{C}=\operatorname{loc}_{\mathrm{K}}\left(C / / \mathrm{s} \mid C \in \mathrm{K}(\operatorname{lnj} \mathrm{R})^{\mathrm{c}}\right) \subseteq \mathrm{T}
$$

since $\mathrm{T}$ is localizing and each $C / / \mathrm{s}$ is in $\mathrm{T}$.

Since $C$ is compactly generated there is a triangle

$$
\Gamma X \rightarrow X \rightarrow \mathrm{L} X \rightarrow
$$

with $\Gamma X \in \mathrm{C}$ and $\mathrm{L} X \in \mathrm{C}^{\perp}$; see 2.7. We have that $\operatorname{Hom}_{\mathrm{K}(\operatorname{Inj} \mathrm{R})}^{*}(D, \Gamma X)$ is $S^{+}$-torsion since $\Gamma X \in \mathrm{C} \subseteq \mathrm{T}$. We have shown above that $X \in \mathrm{T}$. Thus $\mathrm{L} X \in \mathrm{T}$ since $\mathrm{T}$ is triangulated. By definition this means $\operatorname{Hom}_{\mathrm{K}(\operatorname{Inj} \mathrm{R})}^{*}(D, \mathrm{~L} X)$ is $S^{+}$-torsion. Since $D / / \mathrm{s} \in$ $\mathrm{C}$ and $\mathrm{L} X \in \mathrm{C}^{\perp}$, we have that

$$
\operatorname{Hom}_{\mathrm{K}(\operatorname{Inj} \mathrm{R})}^{*}(D / / \mathrm{s}, \mathrm{L} X)=0 .
$$


By 3.4(3) this implies that $\operatorname{Hom}_{\mathrm{K}(\operatorname{Inj} \mathrm{R})}^{*}(D, \mathrm{~L} X)=0$. But since $D$ was an arbitrary compact object and $\mathrm{K}(\operatorname{Inj} \mathrm{R})$ is compactly generated (see 2.3), this shows that $\mathrm{L} X=0$. By the triangle (4.2) this implies that $\Gamma X \cong X \in \mathrm{K}(\operatorname{Inj} \mathrm{R})$ and hence $X$ is an object of $\mathrm{C}=\operatorname{loc}_{\mathrm{K}}\left(C / / \mathrm{s} \mid C \in \mathrm{K}(\operatorname{Inj} \mathrm{R})^{\mathrm{c}}\right)$.

Remark 4.3. The hypothesis that $\mathrm{H}^{n}(M)=0$ for $n \gg 0$ is necessary. Indeed, from the definition of finite injective dimension, recalled in 2.4, if a complex $M$ has finite injective dimension, then $\mathrm{H}^{n}(M)=0$ for $n \gg 0$.

We record the following that was contained in the proof of 4.2 .

Corollary 4.4. Under the assumptions and notation of Theorem 4.2, there is an equality

$$
\operatorname{loc}_{\mathrm{K}}\left(C / / \mathrm{s} \mid C \in \mathrm{K}(\operatorname{Inj} \mathrm{R})^{\mathrm{c}}\right)=\operatorname{loc}_{\mathrm{K}}(\mathrm{i} R) .
$$

Proof. One containment is given by (4.1). For the other direction, note that since the $S$-module $\operatorname{Hom}_{\mathrm{K}(\operatorname{Inj} \mathrm{R})}^{*}(\mathrm{i} R, \mathrm{i} R) \cong \operatorname{Ext}_{R}^{*}(R, R)$ is $S^{+}$-torsion, the proof of 4.2 above shows that $\mathrm{i} R \in \operatorname{loc}_{\mathrm{K}}\left(C / / \mathrm{s} \mid C \in \mathrm{K}(\operatorname{Inj} \mathrm{R})^{\mathrm{c}}\right)$.

Corollary 4.5. Let $R, S$ and $M$ be as in 4.2. Let $s_{1}, \ldots, s_{r}$ be a finite set of homogeneous generators of the ideal $S^{+}$. Set

$$
d:=\max \left\{\operatorname{deg} s_{i} \mid 1 \leq i \leq r\right\} \text { and } l:=\operatorname{lcm}\left\{\operatorname{deg} s_{i} \mid 1 \leq i \leq r\right\} .
$$

Then $\operatorname{inj} \operatorname{dim}_{R} M<\infty$ if one of the following holds:

(1) there exists an integer $n \geq 0$ such that $\operatorname{Ext}_{R}^{j}(M, M)=0$ for all $n \leq j \leq$ $n+d-1 ;$ or

(2) there exists an integer $m \geq 0$ such that $\operatorname{Ext}_{R}^{m l}(M, M)=0$.

Proof. Either condition forces the $S$-module $\operatorname{Ext}_{R}^{*}(M, M)$ to be $S^{+}$-torsion. Indeed, assume that there exists an integer $n$ such that (1) holds. For every $i$ there exists an integer $k_{i}$ such that

$$
n \leq k_{i}\left(\operatorname{deg} s_{i}\right) \leq n+d-1 .
$$

One way to see this is by induction on $n$. Consider the ideal $\left(S^{+}\right)^{k_{1}+\ldots+k_{r}}=$ $\left(s_{1}, \ldots, s_{r}\right)^{k_{1}+\ldots+k_{r}}$ in $S$. It is generated by monomials in the $s_{i}$ of the form $s_{1}^{n_{1}} \ldots s_{r}^{n_{r}}$ for positive integers $n_{i}$ with $\sum n_{i}=\sum k_{i}$. For each such monomial there exists an $i$ such that $n_{i} \geq k_{i}$, else $\sum n_{i}<\sum k_{i}$; applying $\zeta_{M}$ to the monomial, and using that $\zeta_{M}$ is a map of rings, we see that

$$
\begin{aligned}
\zeta_{M}\left(s_{1}^{n_{1}} \ldots s_{r}^{n_{r}}\right) & =\zeta_{M}\left(s_{1}^{n_{1}}\right) \ldots \zeta_{M}\left(s_{i}^{n_{i}}\right) \ldots \zeta_{M}\left(s_{r}^{n_{r}}\right) \\
& =\zeta_{M}\left(s_{1}^{n_{1}}\right) \ldots \zeta_{M}\left(s_{i}^{k_{i}}\right) \zeta_{M}\left(s_{i}^{n_{i}-k_{i}}\right) \ldots \zeta_{M}\left(s_{r}^{n_{r}}\right)=0,
\end{aligned}
$$

since $\zeta_{M}\left(s_{i}^{k_{i}}\right) \in \operatorname{Ext}_{R}^{k_{i}\left(\operatorname{deg} s_{i}\right)}(M, M)=0$. Thus

$$
\left(S^{+}\right)^{k_{1}+\ldots+k_{r}} \operatorname{Ext}_{R}^{*}(M, M)=\zeta_{M}\left(\left(S^{+}\right)^{k_{1}+\ldots+k_{r}}\right) \operatorname{Ext}_{R}^{*}(M, M)=0
$$

and hence $\operatorname{Ext}_{R}^{*}(M, M)$ is $S^{+}$-torsion. By Theorem 4.2 this shows that $\operatorname{inj} \operatorname{dim}_{R} M<\infty$. 
To prove (2) assume that such an $m$ exists. For every $i=1, \ldots, r$, there exists an integer $d_{i}$ such that $d_{i}\left(\operatorname{deg} s_{i}\right)=l$. Letting $\alpha=m\left(\sum_{i} d_{i}\right)$, a similar proof as above shows that $\left(s_{1}, \ldots, s_{r}\right)^{\alpha} \operatorname{Ext}_{R}^{*}(M, M)=0$.

\section{Applications}

In this section, we apply Theorem 4.2 in the two contexts discussed in the introduction.

5.1. Let $R$ be a commutative ring with a presentation

$$
R \cong Q /(\mathbf{f})
$$

where $Q$ is a commutative Noetherian regular ring of finite Krull dimension and $(\mathbf{f})=\left(f_{1}, \ldots, f_{c}\right)$ is a $Q$-regular sequence.

Let $S=R\left[\chi_{1}, \ldots, \chi_{c}\right]$ be the polynomial ring in $c$ indeterminates over $R$, graded by setting $\left|\chi_{i}\right|=2$. For every $X \in \mathrm{K}(\operatorname{lnj} \mathrm{R})$ there is a homomorphism of graded $R$-algebras

$$
\zeta_{X}: S \rightarrow \operatorname{Hom}_{\mathrm{K}(\operatorname{Inj} \mathrm{R})}^{*}(X, X) .
$$

When $X=\mathrm{i} M$ is the injective resolution of a finitely generated $R$-module $M$, so that

$$
\operatorname{Hom}_{\mathrm{K}(\operatorname{Inj} \mathrm{R})}^{*}(X, X) \cong \operatorname{Ext}_{R}^{*}(M, M),
$$

such a map $\zeta_{X}$ may be constructed as in [9, Section 1] using a free resolution of $M$. The process described in [1, Section 1], which replaces free resolutions with injective resolutions, generalizes to arbitrary objects of $\mathrm{K}(\operatorname{Inj} \mathrm{R})$. The results of loc. cit. show that the maps $\zeta_{X}$ satisfy the conditions of a ring of cohomology operators.

By $[6,5.1]$ the $S$-module $\operatorname{Hom}_{\mathrm{K}(\operatorname{Inj} \mathrm{R})}^{*}(\mathrm{i} M, \mathrm{i} M) \cong \operatorname{Ext}_{R}^{*}(M, M)$ is finitely generated when $M$ has finite cohomology over $R$. This was proved first by Gulliksen [11] for modules. It follows from 2.6 that $R$ has Noetherian cohomology over $S$. Restating Theorem 4.2 in this context, we have:

Corollary 5.2. Let $Q$ be a commutative Noetherian regular ring of finite Krull dimension, $(\mathbf{f})=\left(f_{1}, \ldots, f_{c}\right)$ a $Q$-regular sequence and $R=Q /(\mathbf{f})$. For an $R$-complex $M$ with $H^{n}(M)=0$ for all $n \gg 0$, if $\operatorname{Ext}_{R}^{*}(M, M)$ is $S^{+}$-torsion, then $M$ has finite injective dimension.

In the notation of Corollary 4.5 we see that $d=2=l$. Since $R$ is a Gorenstein ring of finite Krull dimension, a module has finite projective dimension if and only if it has finite injective dimension. This gives:

Corollary 5.3. If $M$ is an arbitrary $R$-module such that $\operatorname{Ext}_{R}^{2 n}(M, M)=0$ for some $n \geq 1$ then $M$ has finite projective dimension.

Remark 5.4. In $[2,4.2]$ the same statement is proved for finitely generated modules of finite complete intersection dimension over a Noetherian ring. All finitely generated modules over the ring $R$ have finite complete intersection dimension. However, complete intersection dimension is not defined for non-finitely generated modules, so we have not generalized completely $[2,4.2]$.

5.5. Let $R$ be a Hopf algebra over a field $k$. For two $R$-modules $M, N$ we view $M \otimes_{k} N$ as an $R$-module via the diagonal map $\Delta: R \rightarrow R \otimes_{k} R$. When $M, N$ are injective then so is $M \otimes_{k} N$. For $X \in \mathrm{K}(\operatorname{Inj} \mathrm{R})$ the functor $-\otimes_{k} X$ preserves homotopies of maps. 
Thus there is a functor $-\otimes_{k} X: \mathrm{K}(\operatorname{Inj} \mathrm{R}) \rightarrow \mathrm{K}(\operatorname{lnj} \mathrm{R})$. Viewing $k$ as an $R$-module via the augmentation there is an isomorphism

$$
\varphi_{X}: \mathrm{i} k \otimes_{k} X \stackrel{\cong}{\longrightarrow} X,
$$

see $[8,5.3]$ which proof holds in our more general situation. Thus for each $X$ one gets a map

$$
\eta_{X}: \operatorname{Hom}_{\mathrm{K}}^{*}(\mathrm{i} k, \mathrm{i} k) \rightarrow \operatorname{Hom}_{\mathrm{K}}^{*}(X, X)
$$

that sends $\alpha: \mathrm{i} k \rightarrow \Sigma^{n} \mathrm{i} k$ to

$$
\varphi_{\Sigma^{n} X}\left(\alpha \otimes_{k} X\right)\left(\varphi_{X}\right)^{-1}: X \rightarrow \Sigma^{n} X
$$

One can check that $\eta_{X}$ is a ring map. Let $S$ be the $\operatorname{ring} \operatorname{Ext}_{R}^{*}(k, k) \cong \operatorname{Hom}_{\mathrm{K}}^{*}(\mathrm{i} k, \mathrm{i} k)$. By $\left[14\right.$, (VIII.4.7), (VIII.4.3)] the ring $S$ is graded-commutative and the maps $\eta_{X}$ satisfy the commutativity relations (3.1). Thus setting

$$
S^{\text {even }}:= \begin{cases}\bigoplus_{i \geq 0} \operatorname{Ext}_{R}^{2 i}(k, k) & \text { if } \operatorname{char} k \neq 2, \\ \operatorname{Ext}_{R}^{*}(k, k) & \text { if } \operatorname{char} k=2,\end{cases}
$$

we see that $S^{\text {even }}$ is commutative and is a ring of cohomology operators on $R$.

By the main result of [10], when $R$ is cocommutative and finite-dimensional over $k$, the ring $S$ is Noetherian and $\operatorname{Ext}_{R}^{*}(M, N)$ is a Noetherian $S$-module (via $\eta_{M}$, or equivalently, $\eta_{N}$ ) for all complexes $M, N$ with finite cohomology. The ideal of odd degree elements in $S$ is nilpotent when char $k \neq 2$. Thus when $R$ is a cocommutative finite-dimensional Hopf algebra it has Noetherian cohomology over $S^{\text {even }}$.

Specializing Theorem 4.2 and Corollary 4.5 to this context, and using that $R$ is self-injective, we have:

Corollary 5.6. Let $R$ be a finite-dimensional cocommutative Hopf algebra and $S^{\text {even }}$ the commutative ring defined as above. For an $R$-complex $M$ with $H^{n}(M)=0$ for all $n \gg 0$, if $\operatorname{Ext}_{R}^{*}(M, M)$ is $S^{+}$-torsion, then $M$ has finite injective dimension.

Corollary 5.7. Let $R$ be as above and $M$ an $R$-module. There exists an integer $l$ such that if $\operatorname{Ext}_{R}^{n l}(M, M)=0$ for some $n \geq 1$ then $M$ has finite projective dimension.

\section{Acknowledgments}

This work formed part of my PhD thesis. I would like to thank my advisor Srikanth Iyengar for his support and guidance, Dave Benson for discussing this material with me, and the referee for providing several helpful suggestions including an improved proof of Proposition 4.1. I was partly supported by the NSF Grant DMS 0903493.

\section{References}

[1] L.L. Avramov, Modules of finite virtual projective dimension, Invent. Math. 96(1) (1989), 71101.

[2] L.L. Avramov and R.-O. Buchweitz, Support varieties and cohomology over complete intersections, Invent. Math. 142(2) (2000), 285-318.

[3] L.L. Avramov and H.-B. Foxby, Homological dimensions of unbounded complexes, J. Pure Appl. Algebra 71(2-3) (1991), 129-155.

[4] L.L. Avramov and S.B. Iyengar, Cohomologically Noetherian rings. Preprint.

[5] — Constructing modules with prescribed cohomological support, Illinois J. Math. 51(1) (2007), 1-20 (electronic). 
[6] L.L. Avramov and L.-C. Sun, Cohomology operators defined by a deformation, J. Algebra 204(2) (1998), 684-710.

[7] D. Benson, S.B. Iyengar and H. Krause, Local cohomology and support for triangulated categories, Ann. Sci. Éc. Norm. Supér. (4) 41(4) (2008), 573-619.

[8] D.J. Benson and H. Krause, Complexes of injective $k G$-modules, Algebra Number Theory 2(1) (2008), 1-30.

[9] D. Eisenbud, Homological algebra on a complete intersection, with an application to group representations, Trans. Amer. Math. Soc. 260(1) (1980), 35-64.

[10] E.M. Friedlander and A. Suslin, Cohomology of finite group schemes over a field, Invent. Math. 127(2) (1997), 209-270.

[11] T.H. Gulliksen, A change of ring theorem with applications to Poincaré series and intersection multiplicity, Math. Scand. 34 (1974), 167-183.

[12] M. Hovey, J.H. Palmieri and N.P. Strickland, Axiomatic stable homotopy theory, Mem. Amer. Math. Soc. 128(610) (1997), x+114.

[13] H. Krause, The stable derived category of a Noetherian scheme, Compos. Math. 141(5) (2005), 1128-1162.

[14] S. Mac Lane, Homology, Die Grundlehren der mathematischen Wissenschaften, Bd. 114, Academic Press, New York (1963).

[15] A. Neeman, The connection between the K-theory localization theorem of Thomason, Trobaugh and Yao and the smashing subcategories of Bousfield and Ravenel, Ann. Sci. École Norm. Sup. (4) $25(5)$ (1992), 547-566.

[16] N. Spaltenstein, Resolutions of unbounded complexes, Composit. Math. 65(2) (1988), 121-154.

[17] J.-L. Verdier, Des catégories dérivées des catégories abéliennes, Astérisque (1996), no. 239, xii+253 pp. (1997). With a preface by Luc Illusie, Edited and with a note by Georges Maltsiniotis.

Department of Mathematics, Universität Bielefeld, 33501 Bielefeld, Germany

E-mail address: jburke@math.uni-bielefeld.de

Current address: Department of Mathematics, University of California, los Angeles, CA 90095, USA

E-mail address: jburke@math.ucla.edu 